\title{
Undecidability of Multiplicative Subexponential Logic
}

\author{
Kaustuv Chaudhuri \\ INRIA, France \\ kaustuv. chaudhuri@inria.fr
}

\begin{abstract}
Subexponential logic is a variant of linear logic with a family of exponential connectives-called subexponentials - that are indexed and arranged in a pre-order. Each subexponential has or lacks associated structural properties of weakening and contraction. We show that classical propositional multiplicative linear logic extended with one unrestricted and two incomparable linear subexponentials can encode the halting problem for two register Minsky machines, and is hence undecidable.
\end{abstract}

\section{Introduction}

The decision problem for classical propositional multiplicative exponential linear logic (MELL), consisting of formulas constructed from propositional atoms using the connectives $\{\otimes, 1, \ngtr, \perp, !, ?\}$, is perhaps the longest standing open problem in linear logic. MELL is bounded below by the purely multiplicative fragment (MLL), which is decidable even in the presence of first-order quantification, and above by MELL with additive connectives (MAELL), which is undecidable even for the propositional fragment [5]. This paper tries to make the undecidable upper bound a bit tighter by considering the question of the decision problem for a family of propositional multiplicative subexponential logics (MSEL) [8, 10], each of which consists of formulas constructed from propositional atoms using the (potentially infinite) set of connectives $\{\otimes, 1, \gamma, \perp\} \cup \bigcup_{u \in \Sigma}\left\{!^{u}, ?^{u}\right\}$, where $\Sigma$ is a pre-ordered set of subexponential labels, called a subexponential signature, that is a parameter of the family of logics. In particular, we show that a particular MSEL with a subexponential signature consisting of exactly three labels can encode a two register Minsky machine (2RM), which is Turing-equivalent. This is the same strategy used in [5] to show the undecidability of MAELL, but the encoding in MSEL is different-simpler-for the branching instructions, and shows that additive behaviour is not essential to implement branching. We use the classical dialect of linear logic to show these results. The intuitionistic dialect has the same decision problem because it is possible to faithfully encode (i.e., linearly simulate the sequent proofs of) the classical dialect in the intuitionistic dialect without changing the signature [2].

This short note is organized as follows: in section 2 we sketch the one-sided sequent formulation of MSEL and recall the definition of a $2 R M$. In section 3 we encode the transition system of a $2 R M$ in a MSEL with a particular signature. In section 4 we argue that the encoding is adequate, i.e., that the halting problem for a $2 R M$ is reduced to the proof search problem for this $M S E L$-encoding, by appealing to a focused sequent calculus for MSEL. The final section 5 discusses some of the ramifications of this result.

\section{Background}

\subsection{Propositional Subexponential Logic}

Let us quickly recall propositional subexponential logic (SEL) and its associated sequent calculus proof system. This logic is sometimes called subexponential linear logic (SELL), but since it is possible for 


$$
\begin{aligned}
& \overline{\vdash a, \neg a}_{\text {init }} \frac{\vdash \Gamma, A \quad \vdash \Delta, B}{\vdash \Gamma, \Delta, A \otimes B} \otimes \overline{\vdash 1}^{1} \quad \frac{\vdash \Gamma, A}{\vdash \Gamma, A \oplus B} \oplus_{1} \quad \frac{\vdash \Gamma, B}{\vdash \Gamma, A \oplus B} \oplus_{2} \quad \text { no rule for } 0 \\
& \frac{\vdash \Gamma, A, B}{\vdash \Gamma, A \gamma B} \curvearrowright \frac{\vdash \Gamma}{\vdash \Gamma, \perp} \perp \frac{\vdash \Gamma, A \quad \vdash \Gamma, B}{\vdash \Gamma, A \& B} \& \frac{{ }^{\prime} \Gamma, \top}{\top} \frac{\vdash \Gamma, A}{\vdash \Gamma, ?^{u} A} ? \\
& \frac{\left(u \leq_{\Sigma} \vec{v}\right) \vdash_{\Sigma} ?^{\vec{v}} \vec{A}, C}{\vdash_{\Sigma} ?^{\vec{v}} \vec{A}, !^{u} C} ! \quad \frac{\left(u \in U_{\Sigma}\right) \vdash_{\Sigma} \Gamma}{\vdash_{\Sigma} \Gamma, ?^{u} A} \text { weak } \quad \frac{\left(u \in U_{\Sigma}\right) \vdash_{\Sigma} \Gamma, ?^{u} A, ?^{u} A}{\vdash_{\Sigma} \Gamma, ?^{u} A} \text { contr }
\end{aligned}
$$

Figure 1: Inference rules for a cut-free one-sided sequent calculus formulation of SEL. Only the rules on the last line are sensitive to the signature.

the subexponentials to have linear semantics it is redundant to include both adjectives. Formulas of SEL $(A, B, \ldots)$ are built from atomic formulas $(a, b, \ldots)$ according to the following grammar:

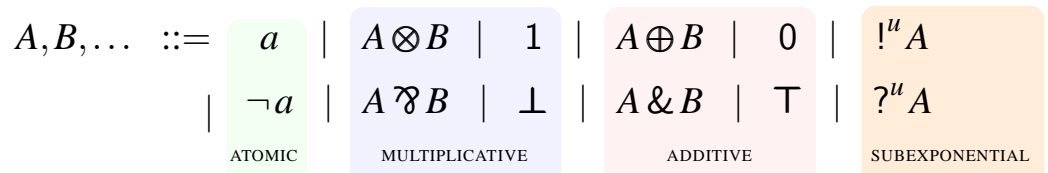

Each column in the grammar above is a De Morgan dual pair. A positive formula (depicted with $P$ or $Q$ when relevant) is a formula belonging to the first line of the grammar, and a negative formula (depicted with $N$ or $M)$ is a formula belonging to the second line. The labels $(u, v, \ldots)$ on the subexponential connectives ! ${ }^{u}$ and $?^{u}$ belong to a subexponential signature defined below. The additive fragment of this syntax is just used in this section for illustration; we will not be using the additives in our encodings. The fragment without the additives will be called multiplicative subexponential logic (MSEL).

Definition 1. A subexponential signature $\Sigma$ is a structure $\langle\Lambda, U, \leq\rangle$ where:

- $\Lambda$ is a countable set of labels;

- $U \subseteq \Lambda$, called the unbounded labels; and

- $\leq \subseteq \Lambda \times \Lambda$ is a pre-order on $\Lambda$ - i.e., it is reflexive and transitive-and $\leq$-upwardly closed with respect to $U$, i.e., for any $u, v \in \Lambda$, if $u \in U$ and $u \leq v$, then $v \in U$.

We will assume an ambient signature $\Sigma$ unless we need to disambiguate particular instances of MSEL, in which case we will use $\Sigma$ in subscripts. For instance, $M S E L_{\Sigma}$ is a particular instance of MSEL for $\Sigma$.

The true formulas of MSEL are derived from a sequent calculus proof system consisting of sequents of the form $\vdash A_{1}, \ldots, A_{n}$ (with $n>0$ ) and abbreviated as $\vdash \Gamma$. The contexts $(\Gamma, \Delta, \ldots)$ are multi-sets of formulas of $S E L$, and $\Gamma, \Delta$ and $\Gamma, A$ stand as usual for the multi-set union of $\Gamma$ with $\Delta$ and $\{A\}$, respectively. The inference rules for SEL sequents are displayed in figure 1 Most of the rules are shared between $S E L$ and linear logic and will not be elaborated upon here. The differences are with the subexponentials, for which we use the following definition.

Definition 2. For any $n \in N$ and lists $\vec{u}=\left[u_{1}, \ldots, u_{n}\right]$ and $\vec{A}=\left[A_{1}, \ldots, A_{n}\right]$, we write $?^{\vec{u}} \vec{A}$ to stand for the context $?^{u_{1}} A_{1}, \ldots, ?^{u_{n}} A_{n}$. For $\vec{v}=\left[v_{1}, \ldots, v_{n}\right]$, we write $u \leq \vec{v}$ to mean that $u \leq v_{1}, \ldots$, and $\left.u \leq v_{n} . \quad\right\lrcorner$

The rule for !, sometimes called promotion, has a side condition that checks that the label of the principal formula is less than the labels of all the other formulas in the context. This rule cannot be used if there are non-?-formulas in the context, nor if the labels of some of the ?-formulas are strictly smaller or incomparable with that of the principal !-formula. Both these properties will be used in the encoding in the next section. The structural rules of weakening and contraction apply to those principal ?-formulas with unbounded labels. 


\subsection{Two Register Minsky Machines}

Like Turing machines, Minsky register machines have a finite state diagram and transitions that can perform I/O on some unbounded storage device, in this case a bank of registers that can store arbitrary natural numbers. We shall limit ourselves to machines with two registers (2RM) a and b, which are sufficient to encode Turing machines.

Definition 3. A $2 R M$ is a structure $\langle Q, *, \mathscr{C}, \rightarrow\rangle$ where:

- $Q$ is a non-empty finite set of states;

- $* \in Q$ is a distinguished halting state;

- $\mathscr{C}$ is a set of configurations, each of which is a structure of the form $\langle q, v\rangle$, with $q \in Q$ and $v$ : $\{\mathrm{a}, \mathrm{b}\} \rightarrow N$, that assigns values (natural numbers) to the registers a and $\mathrm{b}$ in state $q$;

- $\rightarrow \subseteq \mathscr{C} \times I \times \mathscr{C}$ is a deterministic labelled transition relation between configurations where the label set $I=\{$ halt, incra, incrb, decra, decrb, isza, iszb $\}$ (called the instructions).

By usual convention, we write $\rightarrow$ infix with the instruction atop the arrow. We require that every element of $\rightarrow$ fits one of the following schemas, where in each case $q, r \in Q$ and $q \neq r$ :

$$
\begin{aligned}
& \langle q, v\rangle \stackrel{\text { halt }}{\longrightarrow}\langle *,\{\mathrm{a}: 0, \mathrm{~b}: 0\}\rangle \quad(\text { with } q \neq *) \\
& \langle q,\{\mathrm{a}: m, \mathrm{~b}: n\}\rangle \stackrel{\text { incra }}{\longrightarrow}\langle r,\{\mathrm{a}: m+1, \mathrm{~b}: n\}\rangle \\
& \langle q,\{\mathrm{a}: m, \mathrm{~b}: n\}\rangle \stackrel{\text { incrb }}{\longrightarrow}\langle r,\{\mathrm{a}: m, \mathrm{~b}: n+1\}\rangle \\
& \langle q,\{\mathrm{a}: m+1, \mathrm{~b}: n\}\rangle \stackrel{\text { decra }}{\longrightarrow}\langle r,\{\mathrm{a}: m, \mathrm{~b}: n\}\rangle \\
& \langle q,\{\mathrm{a}: m, \mathrm{~b}: n+1\}\rangle \stackrel{\text { decrb }}{\longrightarrow}\langle r,\{\mathrm{a}: m, \mathrm{~b}: n\}\rangle \\
& \langle q,\{\mathrm{a}: 0, \mathrm{~b}: n\}\rangle \stackrel{\text { isza }}{\longrightarrow}\langle r,\{\mathrm{a}: 0, \mathrm{~b}: n\}\rangle \\
& \langle q,\{\mathrm{a}: m, \mathrm{~b}: 0\}\rangle \stackrel{\text { iszb }}{\longrightarrow}\langle r,\{\mathrm{a}: m, \mathrm{~b}: 0\}\rangle
\end{aligned}
$$

For a trace $\vec{i}=\left[i_{1}, \ldots, i_{n}\right]$, we write $\left\langle q_{0}, v_{0}\right\rangle \stackrel{\vec{i}}{\rightarrow}\left\langle q_{n}, v_{n}\right\rangle$ if $\left\langle q_{0}, v_{0}\right\rangle \stackrel{i_{1}}{\longrightarrow} \cdots \stackrel{i_{n}}{\longrightarrow}\left\langle q_{n}, v_{n}\right\rangle$. The $2 R M$ halts from an initial configuration $\left\langle q_{0}, v_{0}\right\rangle$ if there is a trace $\vec{i}$ such that $\left\langle q_{0}, v_{0}\right\rangle \stackrel{\vec{i}}{\rightarrow}\langle *,\{\mathrm{a}: 0, \mathrm{~b}: 0\}\rangle$. (The configuration $\langle *,\{\mathrm{a}: 0, \mathrm{~b}: 0\}\rangle$ will be called the halting configuration.) The halting problem for a $2 R M$ is the decision problem of whether the machine halts from an initial configuration.

The requirement that $\rightarrow$ be deterministic amounts to: $\langle q, v\rangle \stackrel{i}{\rightarrow}\left\langle q_{1}, v_{1}\right\rangle$ and $\langle q, v\rangle \stackrel{j}{\longrightarrow}\left\langle q_{2}, v_{2}\right\rangle$ imply that $i=j, q_{1}=q_{2}$, and $v_{1}=v_{2}$. Note that a trace that does not end with a halting configuration will not be considered to be halting, even if there is no possible successor configuration. It is an easy exercise to transform a given $2 R M$ into one where every configuration has a successor except for the halting configuration.

Theorem 4 ([7]). The halting problem for 2RMs is recursively unsolvable.

\section{The Encoding}

For a given $2 R M$, which we fix in this section, we will encode its halting problem as the derivability of a particular MSEL sequent that encodes its labelled transition system and the initial configuration. We will use the following subexponential signature in the rest of this section. 
Definition 5. Let $\Xi$ stand for the signature $\langle\{\infty, \mathrm{a}, \mathrm{b}\},\{\infty\}, \leq\rangle$ where $\leq$ is the reflexive-transitive closure of $\leq_{0}$ defined by a $\leq_{0} \infty$ and $\mathrm{b} \leq_{0} \infty$.

Definition 6 (encoding configurations). For $c=\langle q, v\rangle$, we write $\mathscr{E}(c)$ for the following $M S E L_{\Xi}$ context:

$$
\underbrace{?^{\mathrm{a}} \neg \mathrm{ra}, ?^{\mathrm{a}} \neg \mathrm{ra}, \ldots, ?^{\mathrm{a}} \neg \mathrm{ra}}_{\text {length }=v(\mathrm{a})}, \underbrace{?^{\mathrm{b}} \neg \mathrm{rb}, ?^{\mathrm{b}} \neg \mathrm{rb}, \ldots, ?^{\mathrm{b}} \neg \mathrm{rb}}_{\text {length }=v(\mathrm{~b})}, \neg q
$$

Definition 7 (encoding transitions). The transitions (1) of the $2 R M$ are encoded as a context $\Pi$ with:

- to represent $\langle q, v\rangle \stackrel{\text { halt }}{\longrightarrow}\langle *,\{\mathrm{a}: 0, \mathrm{~b}: 0\}\rangle$, the elements: $q \otimes \neg \mathrm{h}, \mathrm{h} \otimes !^{\mathrm{a}} \mathrm{ra} \otimes \neg \mathrm{h}, \mathrm{h} \otimes !^{\mathrm{b}} \mathrm{rb} \otimes \neg \mathrm{h}, \mathrm{h} \otimes$ $!^{\infty} 1$ (for some $\mathrm{h} \notin Q$ ):

- to represent $\langle q,\{\mathrm{a}: m, \mathrm{~b}: n\}\rangle \stackrel{\text { incra }}{\longrightarrow}\langle r,\{\mathrm{a}: m+1, \mathrm{~b}: n\}\rangle$, the element $q \otimes\left(\neg r \ngtr ?^{\mathrm{a}} \neg \mathrm{ra}\right)$;

- to represent $\langle q,\{\mathrm{a}: m, \mathrm{~b}: n\}\rangle \stackrel{\text { incrb }}{\longrightarrow}\langle r,\{\mathrm{a}: m, \mathrm{~b}: n+1\}\rangle$, the element: $q \otimes\left(\neg r \ngtr ?^{\mathrm{b}} \neg \mathrm{rb}\right)$;

- to represent $\langle q,\{\mathrm{a}: m+1, \mathrm{~b}: n\}\rangle \stackrel{\text { decra }}{\longrightarrow}\langle r,\{\mathrm{a}: m, \mathrm{~b}: n\}\rangle$, the element: $q \otimes !^{\mathrm{a}} \mathrm{ra} \otimes \neg r$;

- to represent $\langle q,\{\mathrm{a}: m, \mathrm{~b}: n+1\}\rangle \stackrel{\text { decrb }}{\longrightarrow}\langle r,\{\mathrm{a}: m, \mathrm{~b}: n\}\rangle$, the element: $q \otimes !^{\mathrm{b}} \mathrm{rb} \otimes \neg r$;

- to represent $\langle q,\{\mathrm{a}: 0, \mathrm{~b}: n\}\rangle \stackrel{\text { isza }}{\longrightarrow}\langle r,\{\mathrm{a}: 0, \mathrm{~b}: n\}\rangle$, the element: $q \otimes !^{\mathrm{b}} \neg r$; and

- to represent $\langle q,\{\mathrm{a}: m, \mathrm{~b}: 0\}\rangle \stackrel{\text { iszb }}{\longrightarrow}\langle r,\{\mathrm{a}: m, \mathrm{~b}: 0\}\rangle$, the element: $q \otimes !^{\mathrm{a}} \neg r$.

Note that $\Pi$ contains a finite number of elements.

Definition 8 (encoding the halting problem). If $\Gamma$ is $A_{1}, \ldots, A_{n}$, then let $?^{u} \Gamma$ stand for $?^{u} A_{1}, \ldots, ?^{u} A_{n}$. The encoding of the halting problem for the $2 R M$ from the initial configuration $c_{0}=\left\langle q_{0}, v_{0}\right\rangle$ is the $M S E L_{\Xi}$ sequent $\vdash ?^{\infty} \Pi, \mathscr{E}\left(c_{0}\right)$.

Theorem 9. If the $2 R M$ halts from $c_{0}$, then $\vdash_{\Xi} ?^{\infty} \Pi, \mathscr{E}\left(c_{0}\right)$ is derivable.

Proof. We will show that if $c=\left\langle q_{1}, v_{1}\right\rangle \stackrel{i}{\rightarrow}\left\langle q_{2}, v_{2}\right\rangle=d$ (for some $i$ ), then the following MSEL $L_{\Xi}$ rule is derivable:

$$
\frac{\vdash ?^{\infty} \Pi, \mathscr{E}(d)}{\vdash ?^{\infty} \Pi, \mathscr{E}(c)}
$$

This is largely immediate by inspection. Here are three representative cases.

- The case of $i=$ incra: it must be that $v_{2}(\mathrm{a})=v_{1}(\mathrm{a})+1$ and $v_{2}(\mathrm{~b})=v_{1}(\mathrm{~b})$, so $\mathscr{E}(d)=\mathscr{E}(c) \backslash$ $\left\{\neg q_{1}\right\}, \neg q_{2}, ?^{\mathrm{a}}$ ra. Moreover, $q_{1} \otimes\left(\neg q_{2}>?^{\mathrm{a}} \neg \mathrm{ra}\right) \in \Pi$. So:

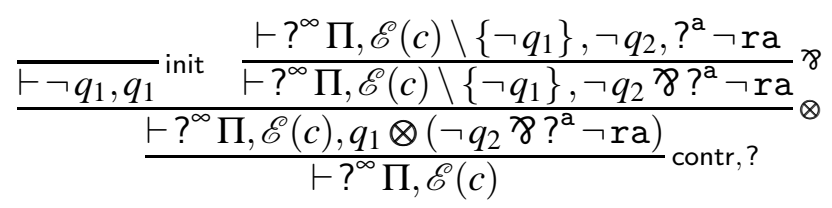

The cases for incrb, decra, and decrb are similar.

- The case of $i=$ isza: it must be that $v_{2}(\mathrm{a})=v_{1}(\mathrm{a})=0$ and $v_{2}(\mathrm{~b})=v_{1}(\mathrm{~b})$. Hence, $\mathscr{E}(d)=$ $\mathscr{E}(c) \backslash\left\{\neg q_{1}\right\}, \neg q_{2}$ and $?^{\mathrm{a}} \mathrm{ra} \notin \mathscr{E}(c) \cup \mathscr{E}(d)$. Moreover, $q_{1} \otimes !^{\mathrm{b}} \neg q_{2} \in \Pi$. So:

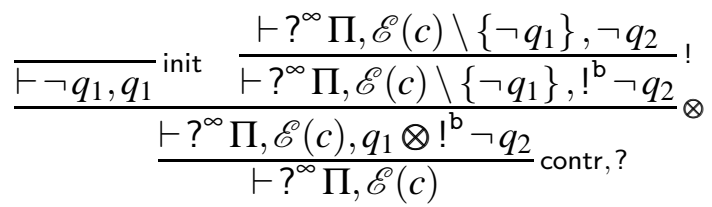

The instance of ! is justified because $\mathrm{b} \leq \infty$ and $\mathrm{b} \leq \mathrm{b}$, and there are no ?-formulas labelled $\mathrm{a}$ or non-? formulas in the sequent. The case of iszb is similar. 
- The case of $i=$ halt. Here, we know that $q_{1} \otimes \neg$ h $\in \Pi$, so:

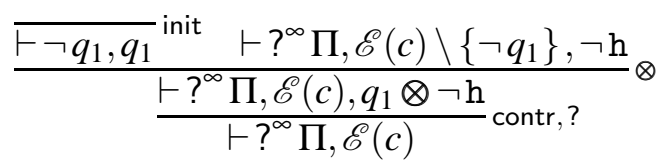

Now, as long as there are any occurrences of $?^{\mathrm{a}}$ ra or $?^{\mathrm{a}} \mathrm{rb}$ in $\mathscr{E}(c)$, we can apply one of the decrementing rules $h \otimes !^{\mathrm{a}} \mathrm{ra} \otimes \neg \mathrm{h}$ or $\mathrm{h} \otimes !^{\mathrm{b}} \mathrm{rb} \otimes \neg \mathrm{h} \in \Pi$. The general case looks something like this, where $\Delta_{\mathrm{ra}}=\{\neg \mathrm{ra}, \ldots, \neg \mathrm{ra}\}$ and $\Delta_{\mathrm{rb}}=\{\neg \mathrm{rb}, \ldots, \neg \mathrm{rb}\}$.

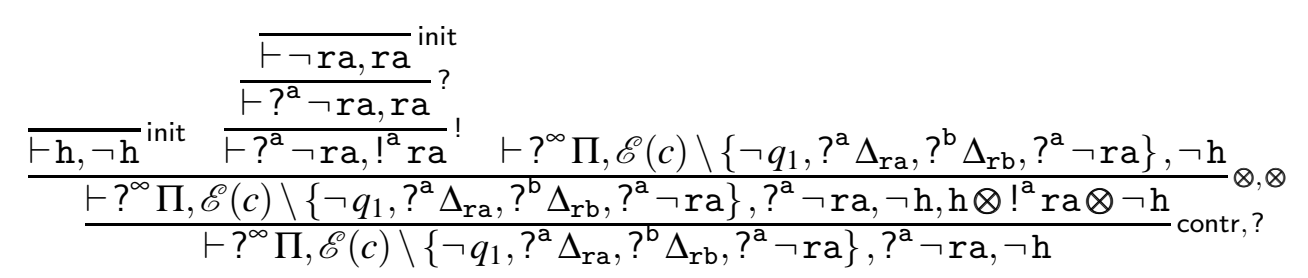

There is a symmetric case for contracting the $\mathrm{h} \otimes !^{\mathrm{b}} \mathrm{rb} \otimes \neg \mathrm{h}$. Eventually, the right branch just becomes $\vdash ?^{\infty} \Pi, \neg \mathrm{h}$, at which point we have:

$$
\begin{gathered}
\overline{\vdash 1}^{1} \\
\vdots \text { weak } \\
\frac{\vdash ?^{\infty} \Pi, 1}{\frac{\vdash \mathrm{h}, \neg \mathrm{h}}{\text { init }} \frac{1 ?^{\infty} \Pi, \neg \mathrm{h}, \mathrm{h} \otimes !^{\infty} 1}{\vdash !^{\infty} 1}} \otimes \\
\frac{\vdash ?^{\infty} \Pi, \neg \mathrm{h}}{} \text { contr }, ?
\end{gathered}
$$

\section{Adequacy of the Encoding via Focusing}

By the contrapositive of theorem 9 , if the sequent $\vdash_{\Xi} ?^{\infty} \Pi, \mathscr{E}\left(c_{0}\right)$ is not derivable, then the $2 R M$ does not halt from $c_{0}$. This gives half of the reduction. For the converse of theorem 9 we need to show how to recover a halting trace by searching for proofs of a MSEL $\Xi$ encoding of a halting problem. The best way to do this is to build a focused proof which will have the derived inference rules in the above proof as the only possible synthetic rules, in a sense made precise below. We will begin by sketching the focused proof system for SEL that is sound and complete for the unfocused system of figure 1 and then show how the synthetic rules for the encoding are in bijection for all instructions (with a small correction needed for halt).

Focusing is a general technique to restrict the non-determinism in a cut-free sequent proof system. Though originally defined for classical linear logic in [1], it is readily extended to many other logics [3, 4. 8]. This section sketches the basic design of a focused version of the rules of figure 1, and omits most of the meta-theoretic proofs of soundness and completeness, for which the general proof techniques are by now well known [3, 6, 13]. To keep things simple, we will define a focused calculus by adding to the unfocused system a new kind of focused sequent, $\vdash \Omega,[A]$, where the formula $A$ is under focus. Contexts written with $\Omega$, which we call neutral contexts, can contain only positive formulas, atoms, negated atoms, and ?-formulas. The rules of the focused proof system for SEL are depicted in figure 2,

Focused sequents are created-reading from conclusion upwards to premises-from unfocused sequents with neutral contexts by means of the rules decide, Idecide, or udecide. In a focused sequent, 


$$
\begin{aligned}
& \frac{\left(\vec{u} \in U_{\Sigma}\right)}{\vdash_{\Sigma} ?^{\vec{u}} \vec{A}, \neg a,[a]}\left[\text { init] } \frac{\left(\vec{u} \in U_{\Sigma}\right) \vdash_{\Sigma} ?^{\vec{u}} \vec{A}, \Omega_{1},[B] \quad \vdash_{\Sigma} ?^{\vec{u}} \vec{A}, \Omega_{2},[C]}{\vdash_{\Sigma} ?^{\vec{u}} \vec{A}, \Omega_{1}, \Omega_{2},[B \otimes C]}[\otimes] \quad \frac{\left(\vec{u} \in U_{\Sigma}\right)}{\vdash_{\Sigma} ?^{\vec{u}} \vec{A},[1]}[1]\right.
\end{aligned}
$$

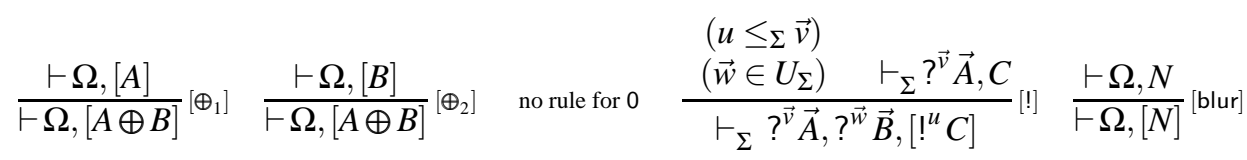

$$
\begin{aligned}
& \text { Rules } \gamma, \perp, \&, T \text { shared with the unfocused system } \\
& \frac{\vdash \Omega,[P]}{\vdash \Omega, P} \text { decide } \quad \frac{\left(u \notin U_{\Sigma}\right) \vdash_{\Sigma} \Omega,[A]}{\vdash_{\Sigma} \Omega, ?^{u} A} \text { Idecide } \quad \frac{\left(u \in U_{\Sigma}\right) \vdash_{\Sigma} \Omega, ?^{u} A,[A]}{\vdash_{\Sigma} \Omega, ?^{u} A} \text { udecide }
\end{aligned}
$$

Figure 2: Inference rules for a focused sequent calculus formulation of SEL.

only the formula under focus can be principal, and the focus persists on the immediate subformulas of this formula in the premises, with the exception of the rule [!]. In the base case, for [init], the focused atom must find its negation in the context, while all formulas in the context must be ?-formulas with unbounded labels. When the focused formula is negative, the focus is released with the [blur] rule, at which point any of the unfocused rules $\{\gamma, \perp, \&, T\}$ of figure 1 can be used to decompose the formula and its descendants further. Eventually, when there are no more negative descendants-i.e., the whole context has the form $\Omega-$ a new focused phase is launched again and the cycle repeats. Note that the structural rules contr and weak of the unfocused calculus are removed in the focused system. Instead, weakening is folded into [init], [!], and [1], and contraction is folded into [®] and udecide. The rules contr and weak remain admissible for either sequent form in the focused calculus.

Theorem 10. The SEL sequent $\vdash \Gamma$ is provable in the unfocused system of figure $\square$ iff it is provable in the focused system of figure 2

Sketch. Straightforward adaptation of existing proofs of the soundness and completeness of focusing, such as [3, 6, 13]. An instance for SEL can be found in [8, chapter 5].

Theorem 11. The $2 R M$ halts from $c_{0}$ if $\vdash_{\Xi} ?^{\infty} \Pi, \mathscr{E}\left(c_{0}\right)$ is derivable.

Proof. We will show instead that the $2 R M$ halts from $c_{0}$ if the sequent $\vdash_{\Xi} ?^{\infty} \Pi, \mathscr{E}\left(c_{0}\right)$ is derivable in the focused calculus, and we will moreover extract the halting trace from such a focused proof. The required result will then follow immediately from theorem 10, since any provable SEL sequent has a focused proof.

Let a focused proof of $\vdash_{\Xi} ?^{\infty} \Pi, \mathscr{E}(c)$ (for $c=\langle q, v\rangle$ ) be given. We proceed by induction on the lowermost instance of udecide in this proof. Note that the $M S E L \Xi$ context $?^{\infty} \Pi, \mathscr{E}(c)$ is neutral; moreover, all the elements of $\mathscr{E}(c)$ are either negated atoms or ?-prefixed negated atoms with bounded labels. So, the only rules of the focusing system that apply to this sequent are Idecide or udecide. However, if we use Idecide, then the premise becomes unprovable, as there is no way to remove an occurrence of $\neg$ ra or $\neg \mathrm{rb}$ from a context that also contains $\neg q$. Thus, the only possible rule will be an instance of udecide, with the focused formula in the premise being one of the $\Pi$. First, consider the case where the focused formula does not contain h, i.e., it corresponds to one of the instructions in $I \backslash\{$ halt $\}$. In each of these cases, the focused phase that immediately follows is deterministic. As a characteristic case, suppose the 
focused formula is $q \otimes !^{\mathrm{b}} \neg r$; then we have:

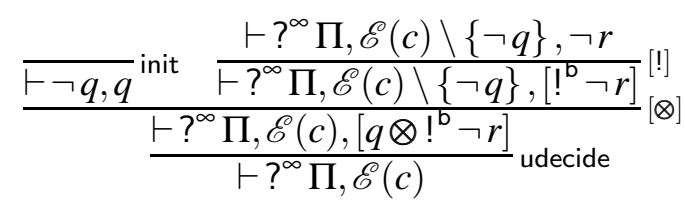

The right premise is now itself neutral and an encoding of a different configuration. We can appeal to the inductive hypothesis to find a halting trace for it, to which we can prepend the instruction isza to get the halting trace from $c$. A similar argument can be used for the other instructions in $I \backslash\{$ halt $\}$.

This leaves just the formulas involving $\mathrm{h}$ for the lowermost udecide. We cannot select any formula but $q \otimes \neg \mathrm{h}$ from $\Pi$, for the derivation would immediately fail because $\mathrm{h} \notin Q$ and there is no $\neg \mathrm{h}$ in $\mathscr{E}(c)$ to use with [init]. So, as the formula selected is $q \otimes \neg \mathrm{h}$, we have:

$$
\frac{\overline{\vdash \neg q,[q]}_{[\text {init }]} \frac{\vdash ?^{\infty} \Pi, \mathscr{E}(c) \backslash\{\neg q\}, \neg \mathrm{h}}{\vdash ?^{\infty} \Pi, \mathscr{E}(c) \backslash\{\neg q\},[\neg \mathrm{h}]}}{\frac{\vdash ?^{\infty} \Pi, \mathscr{E}(c),[q \otimes \neg \mathrm{h}]}{\vdash ?^{\infty} \Pi, \mathscr{E}(c)}} \otimes
$$

The context of the right premise is now neutral, so the only rule that applies to it is udecide. A simple nested induction will show that sequents of this form $\vdash ?^{\infty} \Pi, \mathscr{E}(c) \backslash\{\neg q\}, \neg$ h are always derivable in the focused calculus. Therefore, the trace that corresponds to the configuration $c$ is just the singleton halt.

Corollary 12. The derivability of $M S E L_{\Xi}$ sequents is recursively unsolvable.

Proof. Directly from theorems 4, 9, and 11

\section{Conclusion and Perspectives}

We have given a fairly obvious encoding of a $2 R M$ in a suitable instance of MSEL containing a three element subexponential signature. The encoding of the $2 R M$ halting problem is very similar to that of [5] for MAELL; the main difference is in the encoding of the isz transitions where we can directly check for emptiness of the relevant zone instead of making an additive copy of the world and checking this property in the copy. Additives are therefore not necessary for undecidability.

Yet, this conclusion is not entirely satisfactory. If $M S E L_{\Xi}$ can simulate Turing machines, then it can obviously simulate a theorem prover that implements a complete search procedure for MAELL. Thus, in an indirect fashion, this paper establishes that additive behaviour can be encoded using subexponentials and multiplicatives alone. It would be interesting to build this encoding of additives more directly as an embedding of MAELL — or even just MALL — in MSEL.

This work leaves open the questions of decidability of an arbitrary MSEL with a two-element signature or a one-element signature; the latter is equivalent to the decidability of MELL itself. We also conjecture that the decision problem for an arbitrary MSEL with no unbounded subexponentials is PSPACE-hard, because it is very likely possible to polynomially and soundly encode a MALL sequent in such an MSEL.

Finally, this undecidability result should be taken as a word of caution for the increasingly popular uses of SEL as a logical framework for the encodings of other systems, such as [11, 12]. If one is to avoid encoding a decidable problem in terms of an undecidable one, subexponentials must be used very carefully. 


\section{References}

[1] Jean-Marc Andreoli (1992): Logic Programming with Focusing Proofs in Linear Logic. J. of Logic and Computation 2(3), pp. 297-347, doi:10.1093/logcom/2.3.297.

[2] Kaustuv Chaudhuri (2010): Classical and Intuitionistic Subexponential Logics are Equally Expressive. In: CSL 2010: Computer Science Logic, LNCS 6247, Springer, pp. 185-199, doi:10.1007/ 978-3-642-15205-4_17.

[3] Kaustuv Chaudhuri, Frank Pfenning \& Greg Price (2008): A Logical Characterization of Forward and Backward Chaining in the Inverse Method. J. of Automated Reasoning 40(2-3), pp. 133-177, doi:10.1007/ s10817-007-9091-0.

[4] Chuck Liang \& Dale Miller (2009): Focusing and Polarization in Linear, Intuitionistic, and Classical Logics. Theoretical Computer Science 410(46), pp. 4747-4768, doi:10.1016/j .tcs .2009.07.041.

[5] P. Lincoln, J. Mitchell, A. Scedrov \& N. Shankar (1992): Decision Problems for Propositional Linear Logic. Annals of Pure and Applied Logic 56, pp. 239-311, doi:10.1016/0168-0072 (92) 90075-B.

[6] Dale Miller \& Alexis Saurin (2007): From proofs to focused proofs: a modular proof of focalization in Linear Logic. In J. Duparc \& T. A. Henzinger, editors: CSL 2007: Computer Science Logic, LNCS 4646, Springer, pp. 405-419, doi:10.1007/978-3-540-74915-8_31.

[7] Marvin Minsky (1961): Recursive unsolvability of Post's problem of 'tag' and other topics in the theory of Turing machines. Annals of Mathematics 74(3), pp. 437-455, doi:10.2307/1970290.

[8] Vivek Nigam (2009): Exploiting non-canonicity in the sequent calculus. Ph.D. thesis, Ecole Polytechnique. Available at http://www.nigam.info/thesis/Vivek_Nigam_phd.pdf, pastel:00005487.

[9] Vivek Nigam (2012): On the Complexity of Linear Authorization Logics. In: LICS 2012, IEEE, pp. 511-520, doi:10.1109/LICS.2012.61.

[10] Vivek Nigam \& Dale Miller (2009): Algorithmic specifications in linear logic with subexponentials. In: ACM SIGPLAN Conference on Principles and Practice of Declarative Programming (PPDP), pp. 129-140, doi:10.1145/1599410.1599427.

[11] Vivek Nigam, Carlos Olarte \& Elaine Pimentel (2013): A General Proof System for Modalities in Concurrent Constraint Programming. In: 24th International Conference on Concurrency Theory (CONCUR), LNCS 8052, Springer, pp. 410-424, doi:10.1007/978-3-642-40184-8_29.

[12] Vivek Nigam, Elaine Pimentel \& Giselle Reis (2011): Specifying Proof Systems in Linear Logic with Subexponentials. Electronic Notes in Theoretical Computer Sciience 269, pp. 109-123, doi:10.1016/j .entcs . 2011.03 .009 .

[13] Robert J. Simmons (2014): Structural focalization. ACM Transactions on Computational Logic. 15(3), pp. 21:1-21:33, doi:10.1145/2629678.

Historical note: The undecidability result presented here is from an unpublished paper from 2009, cited as the source of the result in Nigam's Ph.D. thesis from the same year [8, p. 103]. Nigam has also published an indirect proof in [9], using the same strategy and roughly the same encoding, but this version also uses the additive unit $\mathrm{T}$ for halting states and is therefore not strictly in MSEL. 